\title{
Conceptual Integration in Online Interdisciplinary Study: Current perspective, theories, and implications for future research
}

\author{
James L. Morrison \\ University of Oklahoma, USA
}

\begin{abstract}
The University of Oklahoma's College of Liberal Studies was established as an academic unit in 1960, and provides interdisciplinary education to non-traditional students with programs for Bachelor's and Master's degrees in Liberal Studies. In the Fall of 2003, the College offered both degrees in a fully online format, with no student residency requirements. Learners engaged in interdisciplinary studies online are faced with the difficult task of integrating chaotic pieces of information, various ways of knowing, and dissimilar concepts from various disciplines into a coherent and integrated body of knowledge. The purpose of this paper is to discuss the phenomenon of conceptual integration, and the unique challenges and opportunities of providing interdisciplinary study online in the context of conceptual integration.
\end{abstract}

\section{Current Perspective, Theories, and Implications for Future Research}

The intention is to survey the relevant literature, analyze, and synthesize the theoretical and experimental work done across the various areas and disciplines concerning conceptual integration and interdisciplinary online education. Based on current literature, this article provides a theoretical framework for understanding the phenomenon of conceptual integration, and then explores the possibility of enhancing the conceptual integration possibilities in the context of new ways of thinking and learning in the interdisciplinary online education environment. The literature reviewed includes literature that addresses conceptual integration, interdisciplinary education, and online learning. Literature that seems relevant, significant, and provided explanation of definitions is included. The intent is to initiate a discussion of the phenomenon as well as to provide an overview of the research on the subject.

\section{Conceptual Integration as a Phenomenon}

Conceptual integration can be viewed as a cognitive process or as an outcome of the learning process. As a cognitive process, conceptual integration is a background, perhaps even subconscious mental process, which enables one to make meaning from differing concepts that, on the surface, have no readily apparent connection or commonality. Two concepts are integrated into a third concept that contains some properties of both original concepts, but not all of the properties of the two original concepts. Commonalities of the two original concepts provide the 
Morrison Conceptual Integration in Online Interdisciplinary Study:

Current perspective, theories, and implications for future research

basis for an emerging concept that is different from either of the two original concepts. The literature refers to the concepts as mental spaces, and the conceptual integration as the blending of the spaces. The writings of Lakoff (1987), Fauconnier (1994), and Turner (2001) are described below to illustrate the evolution of the concept of conceptual integration as a potential lens through which to frame questions about interdisciplinary online learning.

Lakoff (1987) was convinced that "concepts that are spontaneous, automatic, and unconscious are simply going to have a greater (though less obvious) impact on how we understand everyday life than concepts that we merely ponder (p. 335).” If true, then the online course design that provides interaction with the mental aspects of the student, and elicits spontaneity of thinking, is more powerful than course design that simply presents material that requires reflection.

Lakoff (1987) believed "the idea that people are born with a conceptualizing capacity, seems to be the only plausible way to begin to provide answers for all these questions (p. 335)," concerning conceptualization and the ability of humans to learn new concepts. Lakoff believes humans have an innate ability to blend concepts and this allows them to engage in meaning making. Lakoff also posited that concepts that have been reinforced through experience, especially "perceptual-motor experience," are strong enough to shape our imaginative meaning making and become metaphors by which we understand and view reality.

The early theoretical work of Fauconnier dates from the late 1980s. His work seemed to be concurrent with Lakoff's and Johnson's (1981) development of conceptual metaphor theory, which develops the idea that our "conceptual systems are largely metaphorical (p. 3)." In contrast, Fauconnier, a linguist, explains conceptual integration as a function of language and how we use language to map our mental spaces. Fauconnier (1994) suggests we use descriptions of elements and names to introduce concepts, and linguistic devices such as tense and mood as clues to indicate which mental spaces are the subject of focus (p. xxiii)." The use of the nickname Iron Lady to refer to Margaret Thatcher represents a conceptual integration of; the concept of "iron" as a metal used in construction requiring strength, with the concept of "lady" as a woman who holds political rank. Proper nouns help us by describing two separate mental spaces, yet we understand that Margaret Thatcher is not a woman constructed of steel, but one of strong constitution in the blend of mental spaces. For Fauconnier (1994) it is the:

Lexical information that connects the mental space elements to frames and cognitive models from background knowledge; this information structures the spaces internally by taking advantage of available pre-structured background schemas. Such pre-structured schemas can, however, be altered or elaborated within the constructions under way (p. xxiii).

Fauconnier (1994) used the idea of mental mapping in order to illustrate the cross-domain function of thinking. He describes cross-domain in this way: When we engage in thought mediated with language, we construct mental spaces that are structured, linked, and sometimes projected into other mental spaces. The key aspects of these spaces become visible by means of language. Fauconnier (1997) describes the process of conceptual integration as "integrating partial structures from two separate domains into a single structure with emergent properties within a third domain" (p. 22). In other words, he argues that parts of two separate concepts are blended into a third concept with new attributes contained in the third concept. In interdisciplinary studies, we take portions of two perspectives from different disciplines and create a blended third perspective to solve a problem or understand certain dynamics at work in 
Morrison Conceptual Integration in Online Interdisciplinary Study: Current perspective, theories, and implications for future research

events or societies. We retain portions of the whole, but not all, and both previous perspectives must have commonalities that allow us to blend them.

Fauconnier also began to establish some principles that would enable the examination of cognitive products for conceptual integration.

"Blending is in principle a simple operation, but in practice gives rise to myriad possibilities. It operates on two input mental spaces to yield a third, the blend. The blend inherits partial structure from the input spaces and has emergent structure of its own" (p. 149).

For Fauconnier, partial structure refers to the individual elements of the mental spaces that may or may not be common to the two spaces. In the conceptual integration of Margaret Thatcher as the Iron Lady, the properties of iron are a partial structure of that mental space relating to iron. The properties of English political rank are the partial structures the mental space regarding lady. The property of oxidation is not germane to our blend, yet it is a partial structure of the mental space. The emergent structure of our blend is Margaret Thatcher taking on the property of strength from the iron mental space, along with rank from the mental space of lady.

These principles help us understand the importance of the concept of conceptual integration to the understanding of an interdisciplinary learning environment. Turner's (2001) work serves as an illustration of conceptual integration from the perspective of the social sciences. His view blends the cognitive linguistic perspective of conceptual integration with the anthropologist's perspective of social events and actions. This interdisciplinary view is based on the argument that one cannot examine a concept or an event in isolation from the culture, history, and identity of the persons and society associated with the underlying cognitive operations. Turner (2002) draws on Fauconnier's principles as a foundation. As an illustration, Turner describes how Gertz in his book Deep Play: Notes on the Balinese Cockfight, explicated the social phenomenon of Balinese cockfighting by demonstrating the conceptual blending of event, culture, history, and identity in the underlying cognitive operations (pp. 13-17).

Turner (2002) offers this definition of conceptual integration:

. . . the essence of conceptual integration is its creation of a new mental assembly, a blend, that is identical to neither of its influences and not merely a correspondence between them and usually not even an additive combination of some of their features, but instead a third conceptual space, a child space, a blended space, with new meaning. This new meaning is "emergent" meaning, in the sense that it is not available in either of the influencing spaces, but instead emerges in the blended space by means of blending those influencing spaces ( $\mathrm{p}$. 17).

Here, Turner focuses upon the new meaning that emerges through blended spaces, illustrated by describing the cognitive features of the Balinese cockfight that allow human participants to engage in fighting without personal physical consequences. Turner suggests that cockfighting allows participants to blend their humanity with the beast, their ego with the victorious cock, their social status rising and falling based on the outcome of the fight. Turner expands the ideas of Fauconnier to include mental functions that are a result of "social, cultural, and intentional environments” (p. 46). He extends conceptual integration to include the function of distributed 
Morrison Conceptual Integration in Online Interdisciplinary Study:

Current perspective, theories, and implications for future research

cognition over a period of history through the engagement of "functionally interacting agents and instruments” (p. 46). Conceptual integration, then, encompasses a single individual engaged in blending ideas and mental spaces, or a series of individuals, cultures, and societies engaged in a continuous sequence of blending of concepts and ideas. In fact, powerful blends and metaphors that result may become entangled and embedded in the culture of those groups sharing these mental representations.

\section{Conceptual Integration and Interdisciplinary Study}

Interdisciplinary education provides a rich context for the study of conceptual integration. Complexity, ambiguity, dispersion, and disconnects are adjectives used when discussing interdisciplinary study. Concepts must be analyzed in the context and theoretical framework of the discipline from which they come. Definitions vary according to the literature base and multiple perspectives are embedded in the discipline's community of learners.

Inquiry, approaches to research, and ideas of practice differ among disciplines. Disciplinary work is often considered on a vertical axis measuring the depth of study, and interdisciplinary work may be considered on the horizontal axis for signifying the breadth of study. These two dimensions alone though miss the vital integration of breadth and depth. Synthesis, the integration of concepts, supplies the missing dimension. Synthesis of the deep disciplinary knowledge with the boundary crossing breadth of interdisciplinary study provides the third dimension (Klein, 1996, p. 212).

Conceptual integration is a phenomenon discussed in other disciplines. What follows are descriptions from the fields of Theology, Business, and Educational Psychology. These descriptions help us gain an understanding of the role conceptual integration plays in interdisciplinary study. The significance of conceptual integration as a phenomenon in interdisciplinary study is explained in Moreland's (1999) writing regarding the manner in which theology interacts with other disciplines. Moreland suggests there are a number of ways in which theology and other disciplines interact:

1. The two realms view. Propositions, theories, or methodologies in theology and another discipline may involve two distinct, overlapping areas of investigation.

2. The complementary view. Propositions, theories, or methodologies in theology and another discipline may involve two different complementary, non-interacting approaches to the same reality.

3. The direct interaction view. Propositions, theories, or methodologies in theology and another discipline may directly interact in such a way that either one area of study offers rational support for the other or one area of study raises rational difficulties for the other.

4. The presuppositions view. Theology tends to support the presuppositions of another discipline and vice versa.

5. The practical application view. Theology fills out and adds details to the general principles in another discipline and vice versa, and theology helps one practically apply principles in another discipline and vice versa (p. 8-9). 
Morrison Conceptual Integration in Online Interdisciplinary Study: Current perspective, theories, and implications for future research

These views of interaction help explain why it is critical to find methods that enhance learners' conceptual integration in interdisciplinary courses. They also offer some insights into what activities might be of value in providing an enriched environment. For instance, the use of concepts maps to enhance visualization of connections and relationships, providing access to multiple disciplinary viewpoints and perspectives in course readings. Technology is excellent at providing links to multiple sources of data and information that allow learners access to disciplinary viewpoints. Databases of sociological abstracts and journals provide ready sources of information that may be helpful to a theologian looking at the phenomenon of Church growth, or the organizational aspects of a particular religious institution.

Moreland (1999) also suggests lines of demarcation between disciplines, while strongly defended, are largely philosophical matters (p. 10). If we are to tap in to the exemplary concepts of another discipline, we need those lines to become fuzzy. Conceptual integration provides a vehicle to cross these boundaries.

The field of business provides an example of the application of conceptual integration. Kenney and Leggiere (2003) suggest that building of teams depends heavily on the team having a core story, a story that is "compressed in time and space and that expresses shared values (p. 1)." They further suggest that once the story is developed and stable, the process of conceptual integration can be used to strengthen that story:

When the core story is stable, conceptual blending can help the team derive new, related sub-stories to tackle various business and technical questions, such as the business case or process flow ... through trial and error, the team then maps the elements of one input space to the other. Pairs of connected elements go into the "blended frame." The blended frame is a new story that combines parts of the core story and parts of the standard business case (p.2).

The core story of the team is a conceptual integration of the team member's personal experience and serves to unite the team around an emergent common experience. Ideally, individual stories from the field are continuously merged and internalized as integrated team experience.

Zimmer (1998) explored the use of conceptual integration as a tool of interdisciplinary inquiry. Zimmer posits that evolutionary psychology is a conceptual integration of psychology and evolutionary biology "by tying together psychological phenomena and theories of adaptive function (p.1)”. He further suggests that "conceptual integration has been one distinguishing feature of the natural sciences," and therefore argues that this places evolutionary psychology "on the natural science side of human studies" (p.1). The impact of conceptual integration for Zimmer has "mind-boggling implications," (p. 8) and he believes we have:

... achieved a perspective where the essence of the biblical creation of humans in the image of God is unexpectedly imaged by a proposal in evolutionary psychology on the adaptive function of human awareness of something beyond nature. In achieving this perspective, we recognize a "mythic" or "supernatural" implication to evolutionary science that complements JudeoChristian tradition (p. 8).

Whether you understand or agree with Zimmer, it is apparent that conceptual integration has provided a significant shift in the author's thinking, and that conceptual integration has become a 
Morrison Conceptual Integration in Online Interdisciplinary Study:

Current perspective, theories, and implications for future research

powerful tool of interdisciplinary reflection. Conceptual integration has enabled a reconciliation of the dichotomy of religious belief with science for this author.

While hyper-media may engage us in a new way of learning, Hamilton (2001) suggested that the cognitive architecture of poetry is worthy of investigation because "its language makes us see things in our world with new eyes (p.27).” Metaphor returns us to interdisciplinary study and the significance of conceptual integration in that study. Lakoff (1987) argued that the use of metaphor helps us develop conceptual structure where none existed before. According to Lakoff, "Metaphor provides us with the means for comprehending domains of experience that do not have a pre-conceptual structure of their own in our experience. Comprehending experience via metaphor is one of the great imaginative triumphs of the mind (p.302).” However, Grady, Oakley, and Coulson (1997), concluded that there is a difference between conceptual metaphor theory, "a stable knowledge structure represented in long-term memory" and blending which "seeks to model the dynamic of speakers' on-line representation (p. 120)." This suggest that blending is a cognitive process that is more dynamic and perhaps less stable until rooted in the individuals perspective of reality through metaphor.

\section{Conceptual Integration in the Online Environment}

The online environment should be conducive to conceptual integration and, indeed, requires a high level of conceptual integration to accomplish interdisciplinarity. The online environment supports:

- Through multi-media expanded opportunities for visual and audio reinforcement of ideas and trigger mechanism for reflection

- Non-linear thinking and enables associative reflection

- Adequate opportunity for dialogue using text and language for collaborative activities

- The making of connections and the sustaining of emergent meaning

\section{The Web as a Visual Tool}

The power of visualization is a strength of both technology and the Web. The theoretical framework of conceptual integration provides a powerful argument for the role visual language can play in enhancing conceptual integration. Horn (2001) suggests visual language "has the potential for increasing human bandwidth, the capacity to take in, comprehend, and more efficiently synthesize large amounts of new information (p.1)." Visual language is like having a valve on a fire hose that decreases the flow of water so you can take a drink from that fire hose. Pictures, icons, graphics depicting movement and direction of flow, serve as valves restricting the textual data flow so the human mind can cope with the prodigious amount of data engorging the senses. The constriction through compression actually enhances the human ability to integrate dissimilar concepts and achieve coherence of interdisciplinary knowledge through compression of ideas and thoughts into a visual language.

Muirhead (2002) warns against the proclivity of teachers to focus on content knowledge rather than on the creation of an environment that fosters the use of transferable reflective skills (p.2). For example, Muirhead describes the use of "pictures, cartoons, simulations, or graphics instead of questions" (p.3) as an alternative to the questioning approach as a methodology to enhance 
Morrison Conceptual Integration in Online Interdisciplinary Study:

Current perspective, theories, and implications for future research

dialogue and critical thinking. According to Muirhead, using visual triggers enhances dialogue and reflective thinking, which in turn supports greater integration of the concepts presented in the material. In addition, the use of multi-media as visual language increases the human capacity to process information thus allowing learners to learn more effectively and efficiently. Muirhead focuses on teaching strategies, such as dialogue, as the critical issue in encouraging critical thinking in an online course. Discussion among learners, according to Muirhead, thus becomes the strategy that most promotes "internalization of critical thinking skills and knowledge (p. 6)." Use of visual language and visual triggers incorporated in multi-media enhances both internal and external dialogue. Enhanced dialogue with content or a person leads to a deeper understanding and more thorough integration of concepts. When considering the attributes of technology, it appears this visual language becomes a strength in online courses.

Muirhead (2002) concludes that language is a significant aspect of today's online classes for these classes, "rely heavily on printed materials and teacher created lectures and handouts. Therefore the use of language becomes a focal point for teachers and students because the entire communications process is linked to them" (p.6). Muirhead views conceptual integration from its background in linguistics and the importance of having text. Alfred Bork (2003) also supports the point that "languages are the most powerful learning tools we have," and one of the weaknesses he sees in technology is the "mouse as a computer device (p. 1)." Language may be the most powerful tool we have for learning, but the triggering mechanism of thought and resultant understanding and integration of concepts may be equally important. The visual multi-media aspect of the online environment may serve as the triggering function. Visual language may, through the use of graphics, also enable one to handle more information and increase the value of the concepts integrated.

Cifuentes and Dylak (2003) also speak of the value of "trigger visuals" used to create an emotional response in an attempt to stimulate online discussions. These "trigger visuals" are an important feature of the online environment, and can be incorporated in student prepared multimedia materials in online courses. According to Cifuentes and Dylak, online discussions using these multi-media offerings created by students fostered a constructivist learning environment that included generativity, collaboration, and active engagement. These results of the multi-media learning environment are crucial to the making of meaning for humans, and are interwoven with the following elements of conceptual integration. Fauconnier and Turner (2002) consider the operations of identity, integration, and imagination to be crucial elements "at the heart of even the simplest possible meaning” (p. 6). Based upon this reasoning, it can be argued that conceptual integration should also benefit from the use of "visual triggers" incorporated into the material presented to learners.

\section{Making Connections on the Web Through Hyper-Linking}

Engaged and meaningful learning that seeks out connections and relationships between concepts defines the term deep learning. Rosie (2000) suggests that the attributes of online technology can provide approaches that encourage "deep learning” (p.1). Rosie posits that the Web provides connection opportunities through linking to online learners, providing them the opportunity to seek out and grasp relationships and interconnections between concepts and data. Deep learning involves the bringing together of thesis, antithesis, and synthesis. "What is important is that the synthesis shows not simply progression but a reconceptualisation of the inquiry" (p. 110). Reconceptualisation is key to describing blending in the context of online pedagogies. It appears 
Morrison Conceptual Integration in Online Interdisciplinary Study: Current perspective, theories, and implications for future research

that the phenomenon of conceptual integration, as a background cognitive process, underlies and supports the interconnection of material and the logical organization of material presented.

One can readily see how online study might breakdown into a transmittal of bits of fragmented and meaningless data void of any knowledge building capacity. Fauconnier and Turner (2002) suggest: "The illusion that meaning is transmitted when we send the digitized picture over the Internet is possible, only because there is a brain on each end to handle the construction of meaning” (p.5). This suggests that streams of data and the value of transmitting that information to the online learner lies in the "complex emergent dynamics" that the data or information "triggers in the imaginative mind" (p.6). Content that requires no reflection or activation of the imaginative mind is of no value. The triggering of conceptual integration is of utmost importance to quality synthesis of concepts in online interdisciplinary education. Turner (2001) states:

The theory of conceptual integration is an attempt to provide substance to the intuition that meaning - in individual lives and in cultures - descends through elaborate, perpetual, and distributed processes of modification, inheritance, and selection, to develop all the great richness, diversity, and nuance that characterize cognitively modern human beings and the complex societies they make (p. 151).

It is not enough to transmit information and content, if the goal is synthesis of ideas and concepts and meaning making. Fauconnier and Turner (2002) stress that conceptual integration provides the process by which humans synthesize ideas to make meaning and create knowledge.

The weakness Bork (2003) sees in the technology of online environment is that people begin to think they can deal with the world by pointing and clicking, rather than by thinking. Pointing and clicking seems to have replaced reflection and engagement. It is the cognitive process of conceptual integration that is perhaps the critical component of learning. The mental processes that are required in learning are more than pointing and clicking to navigate through pages.

Integration of information, constructs, and concepts enable one to make sense of and create meaning from divergent streams of data and non-liner links to ideas. Conceptual integration enables one to understand and synthesize ideas drawn from various disciplines and dissimilar bodies of knowledge. A key attribute of online learning is the use of hyper-linking for the construction of knowledge, thus moving from simply gathering data and information to the creation of meaning.

\section{Online Support of Interaction}

In recognition of the need for supporting critical thinking in the online course environment, Muirhead (2002) suggests developing a course structure and teaching style that encourages the engagement of students in higher order thinking skills. Exercising skills such as analysis and synthesis should prove conducive to the underlying mental process of conceptual integration for the learner (p.2). Critical thinking skills that enable thinking in a purposeful, reasoned, goal oriented manner must be applied to concepts and those concepts evaluated in a careful and deliberate manner to reach a conceptual integration outcome that is useful and relevant.

Bullen's (1998) research suggests that, "technology may have attributes that have the potential to facilitate a dynamic and interactive educational experience, making this happen depends on much 
Morrison Conceptual Integration in Online Interdisciplinary Study:

Current perspective, theories, and implications for future research

more than the technology" (p. 17). In fact, Bullen found that factors such as the learners, "previous experience with distance education, cognitive maturity, and experience with interactive learning environments seem to be necessary preconditions for the successful implementation of computer conferencing” (p.17). The use of interactive learning environments seems a strength of online learning, yet Bullen found other non-technological characteristics of learners to be perhaps more important, suggesting that the design of environments may not be the total answer to enhancement of conceptual integration.

\section{Asynchronous Online Environments}

The nature of the online environment is such that communication loses immediacy if asynchronous methods are employed. Asynchronous methods may impede the immediacy because of the time elapsed between communications. Web communications are further hampered by lack of verbal and visual cues. The Web environment enables the user to seek out help from an extraordinary repository of resources, in addition to the immediacy of these resources, time and place independence provide the user with flexibility in their learning schedules and learning spaces. The potential distraction of extraneous material can impede conceptual integration when so many links and resources are available. In addition, the availability of hyperlinks and pop-up screens and messages may interrupt concentration. The sheer magnitude of information may create cognitive overload. Processing of bits of information and incomplete data may hinder the integration of concepts and make it difficult to make meaningful connections between ideas.

Finally, Levine and Sun (2002) suggest, "the Internet is a highly interactive, highly individualized medium," yet distance learning faces several barriers. The most significant barrier is that "academe lacks a pedagogy for using the Internet" (p. 5). Perhaps a good beginning for this new pedagogy is the understanding of the cognitive process of conceptual integration, and the application of the attributes most supportive of enhancing and fostering the blending of a learner's experience and understanding with new information.

\section{Pros and Cons of Online Learning}

As we explore these constructs, we begin to understand the difficulties for the learner engaged in interdisciplinary study delivered in an online format. The speed at which information is transmitted, the myriad connections and jumps across knowledge domains, the non-linear linkages, and time leaps and time compressions that are possible as a learner engages with the interdisciplinary content, provide a fertile learning environment. The speed, the myriad connections and non-linear linkages, can also become overwhelming. The audio-visual presentation can illustrate difficult elements of the concept. Yet, if that presentation is fragmented or exceedingly complex, learners become distracted. The lack of social presence and context may also inhibit understanding or construction of knowledge because there is no dialogue. Online instruction designed by, and for one culture, may result in discontinuity of meaning for another culture.

The reality of the online environment is that interdisciplinary course content and material runs the risk of being fragmented and superficial, making analysis and synthesis difficult for learners. Online education provides a rich environment for the study of the phenomenon of conceptual integration as a cognitive process, and as a desirable outcome of interdisciplinary study. The well 
Morrison Conceptual Integration in Online Interdisciplinary Study:

Current perspective, theories, and implications for future research

designed online learning environment that is conducive to conceptual integration and requires a high level of analysis and synthesis, will pay dividends in the quality of student learning.

\section{Design Considerations for Online Learning}

Design parameters for online interdisciplinary education should include opportunities for learners to engage in the manipulation of language, visual and textual. The environment should provide multi-media presentation to support the presentation of content, visually and aurally as in music, art, and demonstration of skills. However, it is also an important tool for activating reflective engagement, as when new concepts and new perspectives are introduced. Tools, such as Axon2003, enable concept mapping, visual space constructions, and the visual representations of connections, which are crucial to the development and integration of concepts. In addition, the communication capability of the online environment should be exploited to foster dialogue and encourage learners' thinking to be imaginative, generative, and collaborative. The online environment should require students to engage in the crossing of boundaries. This can be done through the use of hyperlinks that lure students into the exploration of multiple perspectives. The online environment can also assist learners in crossing cultural boundaries, by providing for communication with people of diverse cultures, and by bringing new perspectives for the learner's consideration.

\section{Conceptual Integration and Research}

The Cognitive Linguistics literature does not include a large body of data based research. The literature does provide criteria for identifying conceptual integration. Fauconnier's and Turner's present criteria for identifying and analyzing conceptual integration as:

Conditions that are satisfied when two input spaces are blended:

- Cross Space: There is partial mapping of counterparts between the input spaces

- Generic Space: There is a generic space that maps onto each of the inputs. This generic space reflects some common, usually more abstract, structure and organization shared by the inputs and defines the core cross-space mapping between them

- Blend: Inputs are partially projected onto a fourth space, the blend

- Emergent Structure: The blend has emergent structure not provided by the inputs. This happens in three (interrelated) ways

- Composition: Taken together, the projections from the inputs make new relations available that did not exist in the separate inputs

- Completion: Knowledge of background frames, cognitive, and cultural models, allows the composite structure projected into the blend from the inputs to be viewed as part of a larger self-contained structure in the blend. The pattern in the blend triggered by the inherited structures is "completed" into the larger, emergent structure

- Elaboration: The structure in the blend can then be elaborated. This is "running the blend," and consists of cognitive work performed within the blend, according to its own emergent logic 
Morrison Conceptual Integration in Online Interdisciplinary Study: Current perspective, theories, and implications for future research

The central features of blending exemplified by the above criteria are: crossspace mapping, partial projections from inputs, generic space, integration of events, and emergent structure through composition, completion and elaboration (Fauconnier, 1997, p. 151-159).

Several educational researchers have touched on the phenomenon of conceptual integration in their studies of concept development and knowledge construction in online learning. These studies present alternative methods of data based research and measurement of the phenomenon of conceptual integration.

Concept development is a necessary precursor to conceptual integration. McWhirter's (1998) study of conceptual development within the learning cycle provides the methodology to identify and measure conceptual development, and perhaps conceptual integration. Her dissertation "examined concept development and retention within the learning cycle," and investigated "students" concept development mediated by classroom discussions and small cooperative learning groups (p. x). Concept maps were used as the quantitative assessment tools in her study. Students produced the maps and scored using a comparison of their components. A science expert assessed teacher created criterion maps for content. The teacher's map was then used to produce a scoring system template. Multiple coders were used to determine the extent of concept development and retention. Video and audiotapes of the focal group were used to analyze the interaction as a means of triangulation. Concept development was defined as, "important repeatable pattern of two or more distinguished objects, events or situations” (p.39).

McWhirter (1998) used a covariant design for her quantitative analysis, because she discovered that, "reading levels had an effect on students' pre-test scores in both concept mapping and multiple-choice assessment" (p. x). Her research indicated that all three phases of the learning cycle were necessary for conceptual development; however, "individual students showed evidence of concept development and integration at each phase" (p. xi). McWhirter concluded that concept development is individualized, and not all students required each of the three phases of the educational cycle to develop concepts. She also discovered that students who engaged in a high level of dialogue with the instructor, mediated ideas within their small group discussions (p. xi). High levels of dialogue were found to enhance conceptual integration. Concept mapping not only provided a measurement tool, but also appeared to enhance dialogue and understanding of concepts.

Chen (1999) investigated knowledge construction among high school students involved in hypermedia design projects. She determined that evidence of learning could be established by assessing the relationships between concepts the student created, and the organizations of concepts demonstrated by the hypermedia design projects. Chen suggested that the student's struggles with hypermedia design projects engaged them in a new habit of thinking. The four features she describes in terms of this new way of thinking were: typology, associativity, nonlinearity, and abstraction. Chen's observations seem to fit the attributes of the online environment of metaphors indicating conceptual integration to the linguist. Chen also suggests that construction of, and communication of, cognitive products or artifacts actually served to "challenge the learner to engage in higher order thinking” (p. 1). The new way of learning was evaluated by measuring the amount of organization the student exhibited, the depth of the knowledge structures in the concept mapping exercises, and the directionality of the concepts.

This research helps us understand the relationship of conceptual integration in the classroom. We see how concept maps helps students recognize relationships, and helps teachers assess the 
Morrison Conceptual Integration in Online Interdisciplinary Study:

Current perspective, theories, and implications for future research

progression of students' capabilities of developing concepts and engage in higher order thinking. We also see that dialogue has a positive effect on the development of concepts and, by implication, on conceptual integration.

\section{Conceptual Integration and Technology Assisted Deep Learning}

Kanselaar, de John, Andriessen, and Goodyear (2000) suggested that the attributes of the new technology contribute to deep learning and consist of:

1. Multiple Representations: Digital video and animations, graphical representations in distance-time and speed-time graphs - text, speech, and video

2. Technological Mediation: Learning activities that are possible due to the interactive way domain knowledge can be used, computer simulations and discovery learning

3. Computer Mediated Communication: Integrating social and technological mediation in CMC (p. 56)

Kanselaar and colleagues further posited that technology not only has the capability of changing the manner in which people process information, but also the manner in which they represent that information - i.e., through the use of visual language and graphing capabilities of spreadsheet software. They suggested that technology also provides methods by which people can represent information in multiple ways, numbers, and transformation of numbers into graphical representation. In addition, technology has enabled simulation of models and processes. The authors concluded that technology supports collaborative learning through the, "presentation of a task environment to foster student cooperation - joint problem space, ease of data access, intelligent coaching; providing "cooperative tools" - i.e., software that helps one write or a reasoning software tool; by providing a communication facilitator and interface allowing email conferencing or groupware organizational tools; and finally through the use of computer simulation of partner dialogue and problem solving” (p. 67).

\section{Implications and Questions for Future Research}

Young et al. (2000) suggest that from an ecological psychology perspective, the manner in which people learn is explained in terms of the learner's interaction with the properties of their learning environment (p. 148). The online environment has the potential to provide opportunities for interaction and active learning, multi-modal presentation of content, immediacy of communication through email, and group interaction through shareware. Course or environment design, however, must take advantage of these capabilities. Technology also contains inherent barriers, such as including the difficulty of creating a community of learners, lack of expertise on the part of students to fully exploit the hardware, and the dramatic numbers of paths available in navigation, software, or communications portals that may distract learners.

The attributes of technology provide many opportunities to enhance the richness of the information and course content. The visual and audio capabilities of multi-media to provide context and trigger reflection, the non-linear linkage possibilities that produce associative rather than linear thinking, and the capacity to explore issues from a variety of disciplinary resources, all serve to increase the potential for quality learning. 
Morrison Conceptual Integration in Online Interdisciplinary Study: Current perspective, theories, and implications for future research

Future research might investigate questions such as:

1. Can conceptual integration be identified and measured relative to a set of criteria in a quantifiable manner or must we address the issues purely with qualitative methodology?

2. Can conceptual integration be measured in such a way as to determine the extent of its presence and the level of excellence exhibited by the learner in their visual and textual products?

3. Can conceptual integration be enhanced through pedagogical or instructional strategies?

Online interdisciplinary education holds great promise for the learner to reach what Klein (1990) refers to as "the fourth and highest level" (p. 56), or the epitome of interdisciplinary study. At this level, learners have achieved a "conscious attempt to integrate material from various fields of knowledge into a new, single, intellectually coherent entity” (p.56). The understanding of conceptual integration, and the manner in which it is influenced by pedagogical and instructional strategies, is key to enabling students to reach this level of understanding.

\section{References}

Bork, A. (2003). Interactive learning: Twenty years later. Contemporary Issues in Technology and Teacher Education, 2(4). Retrieved April 10, 2003 from: http://citejournal.org/vol2/iss4/seminal/article2.cfm

Bullen, M. (1998). Participation and critical thinking in online university distance education. Journal of Distance Education 13(2). Retrieved May 6, 2003 from: http://cade.icaap.org/vol13.2/bullen.html

Chen, H. (1999). A case study of knowledge construction and knowledge representation in high school students' design of hyper-media documents. An unpublished doctoral dissertation Kansas State University.

Cifuentes, L., and Dylak, S. (2003). Visual Culture: An international telecommunications partnership. Prepared for the AECT Distance Education List Serve discussion. Retrieved April 10, 2003 from: http://www.aect.org/Divisions/trigger.htm

Fauconnier, G. (1994). Mental Spaces: Aspects of meaning construction in natural language. Cambridge: Cambridge University Press.

Fauconnier, G. (1997). Mappings. In Mappings in Thought and Language. Cambridge: Cambridge University Press

Fauconnier, G., and Turner, M. (2002). The Way We Think: Conceptual blending and the mind's hidden complexities. New York: Basic Books.

Grady, J. Oakley, T., and Coulson, S. (1997). Blending and metaphor. In R. Gibbs Jr. and G. Steen (Eds.) Metaphor in Cognitive Linguistics. Selected papers from the Fifth 
Morrison Conceptual Integration in Online Interdisciplinary Study: Current perspective, theories, and implications for future research

International Cognitive Linguistics Conference. Amsterdam, July 1997. Amsterdam: John Benjamins Publishing Company.

Hamilton, C. (2001). Opaque Enigmas: Mind, body, and metaphor in W. H. Auden's Poetry. Unpublished doctoral dissertation University of Maryland at College Park.

Horn, R. (2001). Visual language and converging technologies in the next 10-15 years (and beyond). A paper prepared for the National Science Foundation Conference on Converging Technologies (Nano-Bio-Info-Cogno) for Improving Human Performance Dec. 3-4 2001.

Kanselaar, G., de John, T., Andriessen, J., and Goodyear, P. (2000). New Technologies. R. Simons, J. van der Linden, and T. Duffy. (Eds.) New Learning. London: Klewar Academic Publishers.

Kenney, C., and Leggiere, P. (2003). Across the Chasm. Intelligent Enterprise. Retrieved March 7, 2003 from: http://www.intelligententerprise.com/030320/605feat3_2.shtml

Klein, J. (1990). Interdisciplinarity: History, theory, practice. Detroit, MI.: Wayne State University Press.

Klein, J. (1996). Crossing Boundaries: Knowledge, disciplinarities, and interdisciplinarities. Charlottesville, VA.: University Press of Virginia.

Lakoff, G., and Johnson, M. (1981). Metaphors we live by. Chicago, IL.: University of Chicago Press.

Lakoff, G. (1987). Women, Fire, and Dangerous Things: What categories reveal about the mind. Chicago, IL.: University of Chicago Press.

Levine, A., and Sun, J. (2002). Barriers to distance education. Sixth in the series Distributed education: Challenges, choices, and a new environment. Commissioned by the American Council on Education and EDUCAUSE.

McWhirter, L. (1998). Conceptual development and retention within the learning cycle. An unpublished doctoral dissertation University of Oklahoma.

Moreland, J. (1999). Integration and the Christian scholar. Paper presented to the Christian Scholarship Conference, Ohio State University, Columbus. October. Retrieved March 7, 2003 from: http://www.leaderu.com/aip/docs/moreland2b.html

Muirhead, B. (2002). Integrating critical thinking skills into online classes. USDLA Journal. 16(11), 1 - 7. Retrieved April 10, 2003 from: http://www.usdla.org/html/journal/NOV02 Issue/article03.html

Rosie, A. (2000). Online pedagogies and the promotion of "deep learning." Information Services and Use. IOS Press. $110-116$. 
Morrison Conceptual Integration in Online Interdisciplinary Study: Current perspective, theories, and implications for future research

Simons, R., van der Linden, J., and Duffy, T., (2000). New Learning. London: Klewar Academic Publishers.

Turner, M. (2001). Cognitive Dimensions of Social Science. New York: Oxford Press.

Young, M., Barab, S., and Garrett, S. (2000). Agent as detector: An ecological psychology perspective on learning by perceiving-acting systems. In D. Jonassen \& S. Land. (Eds.) Theoretical foundations of learning environments. Mahwah, NJ.: Lawrence Erlbaum Associates.

Zimmer, J. R. (1998). Evolutionary psychology challenges the current social sciences. In Evolution Dialogue, The American Scientific Application. Retrieved March 7, 2003 from: http://www.asa3.org/ASA/topics/Evolution/PSCF9-98Zimmer.html

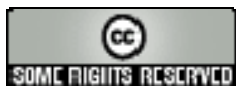

